ÉGYPTE monde arabe

\section{Égypte/Monde arabe}

29 | 1997

Mélanges

\title{
Féminisme et orientalisme au miroir francophone d'Out-El-Kouloub (1892-1968)
}

Julia Madœuf

\section{(2) OpenEdition \\ Journals}

Édition électronique

URL : https://journals.openedition.org/ema/270

DOI : $10.4000 /$ ema. 270

ISSN : 2090-7273

Éditeur

CEDEJ - Centre d'études et de documentation économiques juridiques et sociales

Édition imprimée

Date de publication : 31 mars 1997

Pagination : 101-114

ISSN : 1110-5097

Référence électronique

Julia Madœuf, « Féminisme et orientalisme au miroir francophone d'Out-El-Kouloub (1892-1968) »,

Égypte/Monde arabe [En ligne], 29 | 1997, mis en ligne le 08 juillet 2008, consulté le 07 juillet 2022.

URL : http://journals.openedition.org/ema/270 ; DOI : https://doi.org/10.4000/ema.270

Ce document a été généré automatiquement le 7 juillet 2022.

Tous droits réservés 


\title{
Féminisme et orientalisme au miroir francophone d'Out-El-Kouloub (1892-1968)
}

\author{
Julia Madœuf
}

1 En 1934, la revue L'Égyptienne fait paraître la critique d'un premier texte, Au hasard de la pensée d'Out-El-Kouloub, femme de lettres égyptienne d'expression française ${ }^{1 .}$ Aujourd'hui totalement oubliée en France (les sept écrits publiés dans ce pays sont épuisés), elle a connu son heure de gloire dans les années cinquante. En 1961, s'étant vu confisquer les biens mobiliers de la confrérie soufie qu'elle dirige dans le centre du Caire, "sa famille décida de ne pas rentrer en Égypte tant que le régime nassérien serait en place $»^{2}$. Cette année 1961 coïncide avec la parution du dernier roman d'OutEl-Kouloub (elle décède en Europe en 1968). Contrairement à Andrée Chédid et à Albert Cossery, ses pairs littéraires et ses frères $\mathrm{d}^{\text {'exil }}{ }^{3}$, Out-El-Kouloub ne concevait pas que la création littéraire puisse voir le jour hors de l'Égypte ${ }^{4}$.

«Grande dame»; «romancière de talent »; " autre géniale et indiscrète George Sand »; "femme étonnante par l'ampleur et l'éclectisme de sa culture » autant de formules relevées ici et là sur Out-El-Kouloub, qui, outre leur attrait, intriguent quand on considère son anonymat actuel. C'est ainsi dans le contexte historique, féministe, linguistique et littéraire du pays qu'il conviendra d'appréhender la richesse de cette œuvre et l'originalité de son auteur, dont le moins que l'on puisse dire est qu'elle réside dans un réalisme aux prises avec les représentations orientalistes.

De la francophonie à la naissance d'une littérature

3 A la différence de l'anglais, langue de l'occupant du monde des affaires depuis 1882, le français devient, à partir de la fin du xix siècle, la langue de l'élite égyptienne, l'aristocratie puis la haute bourgeoisie, mais également, dans les années cinquante, celle des classes moyennes qui forment alors la société cosmopolite du pays.

Il y a beaucoup de Libanais en Égypte. Beaucoup d'Européens aussi (...) Italiens, Grecs, Maltais, aussi bien que Français de France - et, d'un groupe à l'autre, ils s'entretiennent en français. Ils rejoignent ainsi les Égyptiens de naissance, épris d'une langue qu'ils associent aux étapes les plus marquantes de leur prise de 
conscience nationale : le passage de Bonaparte avec ses savants, Champollion et le déchiffrement des hiéroglyphes, Lesseps et le canal de Suez. Ils ont fréquenté les écoles françaises, ils ont des journaux, des revues, des éditions en français. De ce milieu cosmopolite naît une littérature désireuse de s'éveiller à l'Orient. ${ }^{5}$

Plusieurs paramètres de la francophonie en Égypte sont abordés ici : celui du français comme langue de communication entre différentes minorités installées dans le pays, celui de la dimension culturelle acquise, enfin celui de la francophilie locale. Mais d'autres aspects plus fondamentaux restent à relever.

5 Ainsi, la prise de conscience nationale connaît son apogée au moment de l'occupation britannique. De ce nationalisme émergent les débuts d'une expression féminine sur l'arène publique, espace que les femmes revendiquent à une époque où la réclusion domestique est encore la norme des citadines des classes moyennes et supérieures, bien que ce soit une norme en cours d'érosion. Le problème de l'aliénation d'une culture, qui est alors clairement posé, cristallise celui de l'aliénation d'un sexe. L'affirmation d'une conscience nationale va être à l'origine des premières revendications féminines. Cette émergence d'un discours public des femmes a rendu possible une production écrite, puis la naissance d'une littérature « instauratrice », " expérimentale ». Dans ce courant prend place la femme écrivain égyptienne d'expression française, Out-El-Kouloub El Demerdachiah (1892-1968). Appartenant à l'aristocratie, elle se consacre à la littérature ${ }^{6}$, reçoit écrivains égyptiens et français; à l'instar d'autres femmes intellectuelles du pays, son salon littéraire est assez prisé.

$6 \mathrm{Au}$ début du siècle, la littérature féminine, d'expression française, féministe et nationaliste pour une bonne part, relève clairement de la transgression. Paradoxalement, il semble que les autorités ne s'en inquiètent pas car l'usage du français est associé aux relations avec la Cour, à la distinction sociale, mais aussi et d'abord au pouvoir en place. C'est ainsi, entre autres, qu'Henri Peyre constate que « des revues souvent plus ferventes, plus éprises d'avant-garde que celles de France voient le jour et publient les écrits de [cette jeune élite] ${ }^{7}$. D'autre part, l'usage du français offre la possibilité de conquérir d'autres publics que les autochtones, dessein essentiel des auteurs qui s'attachent à donner une nouvelle Description de l'Égypte et de ceux qui veulent se rattacher à des écoles littéraires françaises. Le lectorat visé est occidental, le phénomène de l'apport littéraire doit s'inverser, de l'Égypte vers la France.

Faire connaître l'Orient arabe dans sa vérité et sa profondeur, en en donnant des

images surgies de l'intérieur même de cette société, tel est le projet (parfois

clairement explicité) qui anime de nombreux écrivains francophones d'Égypte. ${ }^{8}$

La forme romanesque : prétexte à une nouvelle Description de l'Égypte

7 Cependant, la francophonie en Égypte est plus problématique qu'il n'y parait, dès lors qu'on aborde la production écrite et les usages fort divers que les écrivains - et les femmes-auteurs a fortiori - font de cette langue. Cette aventure linguistique renvoie bien évidemment aux rapports complexes entre une littérature et une société, dans un espace et à un moment donnés. Dans ce contexte précis, la production littéraire est étroitement liée à la situation historique, aux bouleversements, aux chocs culturels. La corrélation de ces éléments avec l'expression féminine francophone est nette, comme si une histoire des femmes, de l'Égypte, se dessinait à travers le texte littéraire.

8 Les œuvres s'éclairent dans leur relation à une autre langue, une autre religion, une esthétique dissemblable, un imaginaire différent. Et cet éclairage varie d'un auteur à un autre. Chaque écrivain se positionne .par rapport à l'idée qu'il se fait de la littérature occidentale, les influences étant multiples et diversement reçues. Ici, intervient le 
problème du «modèle » ou de l'« anti-modèle » littéraire occidental. Les récits de voyage sur l'Égypte font ainsi partie du patrimoine culturel de cette élite qui s'exprime et écrit en français. Ils définissent même la thématique des premiers écrits féminins égyptiens qui vont s'attacher, dans un même temps, à satisfaire les amateurs de récits de voyage et à corriger les inexactitudes et les préjugés nombreux, voire les fabulations concernant l'Orientale, la musulmane, l'Égyptienne.

9 La.femme-auteur veut «se dire » par elle-même, "s'illustrer». Aussi vont d'abord dominer, dans le contexte littéraire, l'expérience du vécu et du vu; la perception du réel. L'œuvre a une fonction, celui du pouvoir de révélation, elle ne se contente plus de " dire un espace ", comme cela a été le cas pour les premiers écrivains francophones du pays, et même si certains se sont également prêtés à la rectification des vieux mensonges exotiques. Les femmes vont "dire leur espace", notamment à travers la presse féminine. Ainsi, il y a au minimum une thématique commune, récurrente et forte, qui se dégage de ces textes, celle de la condition féminine, écrite par des femmes d'origine et de confession diverses, mais qui partagent un même espace culturel : « La francophonie ne désigne pas simplement une réalité linguistique, géographique et sociale mais également le sentiment d'appartenir à une même communauté »". Pour une partie des femmes égyptiennes, il semble que le français représente, au début du siècle, les principes progressistes, qu'il symbolise l'émancipation, puis la liberté. C'est à travers cette représentation qu'Out-El-Kouloub façonne le personnage de l'une de ses héroïnes :

- Créature perverse ! s'écria-t-il, tu ne retiens que les opinions les plus néfastes. Voilà à quoi aboutit l'instruction qu'on te donne ! J'ai deux filles : je jure qu'elles ne sauront jamais lire! Je dirai à la grand-mère qu'elle veille sur toi, car tu es en danger.

- Je ne resterai pas avec ma grand-mère, déclarai-je en le regardant en face. J'apprends le français ; quand je le saurai bien j'irai à Paris. ${ }^{10}$

Le français, ou les chemins de traverse de l'expression

10 Mais au niveau du texte littéraire le français ne revêt-il pas d'autres aspects? Le français comme langue non maternelle peut signifier une plus grande liberté, permettre une distance. C'est un leitmotiv chez les auteurs qui adoptent une langue d'expression écrite différente de leur langue maternelle. Comme s'il y avait une langue " de l'intérieur " et une autre "de l'extérieur ». Lors de sa première déclaration en février 1925, la revue L'Égyptienne est explicite sur ce choix linguistique:

En fondant cette revue dans une langue qui n'est pas la nôtre, mais qui en Égypte comme ailleurs est parlée par toute l'élite, notre but est double : faire connaître à l'étranger La Femme Égyptienne, telle qu'elle est de nos jours (...) et éclairer l'opinion publique européenne sur le véritable état politique et social de l'Égypte."

11 Cette revue est l'exemple type d'une publication proprement «égyptienne », en langue française, s'adressant à l'élite sociale. En ce qui concerne la production littéraire, l'objectif d'Out-El-Kouloub rejoint celui de la revue. D'une part, il s'agit de souligner l'évolution du statut de la femme en Égypte, d'aborder les problèmes qu'elle rencontre ; d'autre part, d'invalider les raccourcis tellement fréquents sur la situation de la femme dans l'Islam. .

12 Mais le choix du français permet également d'échapper à un autre ordre de difficultés : celui auquel sont confrontés les écrivains de langue arabe chaque fois qu'ils tentent de dire un réel au travers d'une langue sacralisée (l'arabe classique), par définition réfractaire à ce dernier et à son imitation. En Égypte, il faudra attendre Naguib 
Mahfouz pour assister à la naissance du roman réaliste en «langue tierce ", fruit de la combinaison entre l'arabe classique et l'arabe parlé. Ce dernier est le premier écrivain égyptien qui a su s'imposer au moyen de cette langue désacralisée et de ce genre (le roman), alors presque tout nouveau en Orient. Le problème se pose avec plus d'acuité au début du siècle et persiste pour la génération d'Out-El-Kouloub.

Dans ce contexte, il devient aisé de voir comment la tradition orale féminine est a fortieri exclue de la langue classique : « Au Liban, comme dans tous les pays du Machreq et du Maghreb, l'arabe [classique] est la langue officielle de l'État mais il n'est la langue maternelle de personne. $\gg^{12}$ La dichotomie entre dialecte et arabe classique (fushâ) est plus que jamais d'actualité aujourd'hui, comme ont pu en témoigner récemment les nombreux débats des Belles Étrangères, manifestation littéraire consacrée à l'Égypte en novembre-décembre 1994. Les femmes écrivains alors présentes, ont expliqué la difficulté accrue pour elles, de rompre avec le classicisme de la langue, rupture bien plus mal vue quand l'auteur est une femme, puisque les lecteurs attendent d'elle une parfaite reproduction de la tradition littéraire, conforme à l'héritage masculin. En écrivant en français, et en parvenant à combiner romance et réalisme, Out-El-Kouloub transmet paradoxalement cet héritage féminin oral (elle dédie Harem à sa mère, « qui aurait aimé cette évocation de la vie qu'elle a connue »).

Paradigmes de polémiques

Si Out-El-Kouloub a échappé à cette polémique en choisissant d'écrire en français, elle a été en butte à d'autres attaques. La théorie selon laquelle l'usage d'une autre langue constitue la perte de soi, de sa culture, est alors d'actualité. Taha Hussein s'exprime sur cette question en évoquant précisément « le cas Out-El-Kouloub » :

II est de mon devoir de présenter aux Égyptiens ce phénomène franco-égyptien, afin qu'ils apprécient son talent qui afflige autant qu'il réjouit. La réjouissance provient du fait que son œuvre invite à la connaissance des Égyptiens et de leurs revendications. Aussi est-ce un honneur pour nous, que nos poètes et écrivains soient l'objet d'estime d'éminents milieux littéraires étrangers. Toutefois, il est déplorable que les œuvres d'Out-El-Kouloub parviennent [aux Égyptiens] par le moyen de la traduction, car c'est à la langue arabe que revient le monopole des œuvres de ses citoyens."

15 Si la critique de Taha Hussein souligne la position biculturelle délicate de la romancière, elle pose également la question plus générale de l'appartenance des auteurs étrangers d'expression française; comment et où les cataloguer? Mais la francophonie en Égypte porte-t-elle réellement ombrage à la langue nationale ? N'estelle pas plutôt le lieu d'une aventure culturelle originale ? Le français d'Out-El-Kouloub veut représenter un combat pour la diversité, une lutte contre les risques de "dépersonnalisation" des individus et d'uniformisation des cultures. Bien plus fondamentalement, le problème que pose ou révèle le choix du français, c'est celui du rapport de l'écrivain égyptien à son lectorat et à sa société :

C'est que l'intellectuel, n'étant pas en contact avec la totalité de sa société peut alors recourir indifféremment à l'arabe classique ou à une langue étrangère puisque de toutes façons, il s'adresse uniquement à lui-même ou à ses frères intellectuels."

Effectivement, d'une part le problème de l'analphabétisme aggrave et amplifie ce genre de situation, d'autre part les inégalités sociales sont grandes et les rapports entre les différentes classes presque inexistants à cette époque (Out-El-Kouloub publie son premier écrit en 1934). Cependant, si ces textes ne peuvent s'adresser à un lectorat égyptien populaire, en revanche, l'auteur utilise un français conventionnel et un genre 
littéraire qui la mettent en mesure de s'attacher un vaste public occidental. Cependant, ces recours ne la mettront pas à l'abri chez elle, puisque lors de la parution des Trois Contes de l'amour et de la mort en 1940, son rapport à l'Occident sera épinglé dans la presse égyptienne d'expression française. Une critique verra dans l'évocation de jeunes parisiennes un peu trop émancipées, une généralisation sur les Occidentales, et elle lui rappellera l'anecdote de cet anglais qui, débarquant en France et apercevant une rousse, retournera dans son pays en affirmant que toutes les Françaises sont rousses...

Madame Out-El-Kouloub, qui est mieux que quiconque au courant des traditions et des mœurs égyptiennes, a le désir fort louable de les rendre familières au public de langue française. Mais elle ne paraît pas aussi bien placée pour faire connaître l'Occident à ses compatriotes. On a en effet l'impression en lisant Zaheira ${ }^{15}$ que ses regards de voyageuse n'ont pas été très profonds. Peut-être même n'ont-ils pas été exempts d'une certaine prévention qu'on s'étonne de rencontrer chez une femme à

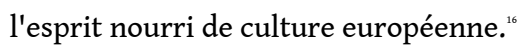

17 Comme cet extrait le révèle, la question toujours actuelle des relations entre l'Orient et l'Occident est épineuse et les œuvres littéraires, leurs personnages de fiction, n'échappent pas à la loupe d'observateurs de tous camps.

Difficultés d'une démarche, inéluctable nostalgie

Out-El-Kouloub rappelle souvent son attachement à sa terre natale, sa religion, comme si elle devançait certaines critiques, prévoyait les attaques sur une langue dont l'expression contribuerait, si elle n'était pas vigilante, à affaiblir aux yeux des lecteurs et des « autres » son appartenance à l'Égypte, à l'Islam.

Ainsi, la thématique de l'enracinement est la substance constitutive de La Nuit de la destinée, et son principal ressort.

Grâce à ce que je tiens de lui [son père], j'ai pu parcourir le monde et me pencher sur les plus brillantes civilisations, sans jamais perdre la croyance de mon enfance qui est sans cesse allée se tonifiant. ${ }^{17}$

$20 \mathrm{Au}$ delà du sentiment religieux, elle paraît craindre pour ses compatriotes la paupérisation, voire la perte, de la mémoire collective, essentiellement composée à partir de l'héritage islamique, et pour elle colonisée en partie par l'Occident. Les souvenirs de l'écrivain, matière à regrets, sont « accrochés à la foi de [ses] pères ». Ce même patriarcat, dont la responsabilité est engagée dans les romans, définit " l'impossible bonheur » des héroïnes, lesquelles, sous sa coupe et celle d'Out-El-Kouloub, ne concrétisent jamais leurs aspirations, même les plus modestes. Tous les romans s'achèvent en drame, en tragédie. Cette méthodique application à ne pas donner d'autre issue à ces personnages de femmes ne peut représenter exclusivement les préférences narratives de l'auteur, elle symbolise plutôt le désenchantement d'une génération sacrifiée.

21 En restituant une Égypte traditionnelle et familière, elle entend lutter contre les risques d'acculturation du pays, qu'elle trouve accrus par l'occupation anglaise. Dans un même temps, la société, sans perdre de vue ces prérogatives culturelles, doit s'affranchir de certaines de ses traditions pour permettre la libération de la femme. Cette volonté conciliatrice délicate, se rapproche de ce que Jean-Jacques Luthi définit comme «le désir de rénovation et de participation au rythme du monde actuel (...) désir [qui] va de pair avec celui de garder, dans son intégrité, le legs du passé. $»^{18}$ Les personnages illustrent la difficulté de la démarche. Dans Ramza, la situation de l'héroïne révoltée, confrontée à la loi patriarcale, est assimilée à celle du peuple 
égyptien sous domination britannique. Le père de Ramza, traducteur à la Cour, voit le Khédive Abbas se rallier à la cause de sa fille, Lord Cromer à la sienne. Et si Ramza parvient à se libérer des contraintes les plus lourdes, il n'en est pas de même pour tous les personnages, qui figurent la diversité des comportements. Les protagonistes d'OutEl-Kouloub, en majorité des femmes, ne sont pas tous des champions de l'indépendance, ils personnifient plutôt les réalités historiques et sociales de l'époque, des époques. La romancière met en scène des êtres humains bien plus que des idées, même si elle place l'enfance et l'adolescence de ses personnages au centre d'une nécessaire reconstruction de la société. De plus, ces jeunes filles, ces femmes, sont issues de tous les milieux: aristocratie (Ramza), bourgeoisie (Hefnaoui le magnifique, Zanouba), milieu rural (Trois contes de l'amour et de la mort), artistique (Le Coffret hindou). Out-El-Kouloub a eu la charge d'un vaste domaine agricole, c'est ainsi qu'elle a été en contact avec d'autres classes sociales. Cette activité et ces rencontres représentent une double originalité. La première tranche par rapport à l'habituelle responsabilité masculine sur la propriété, et les deuxièmes s'opposent au cloisonnement social qui règne alors; opposition qui est l'une des composantes du mouvement d'expression féminine.

Aujourd'hui, ce que l'on reproche au mouvement d'expression féminine, dans son ensemble, et en particulier à l'UFE', qui, dans son efficacité le symbolise, c'est de n'avoir concerné que les émettrices du message, c'est-à-dire un groupe restreint de bourgeoises et d'aristocrates, et de n'avoir laissé aucune trace profonde. ${ }^{20}$

On a également fait grief à la romancière d'une forme de superficialité, notamment sur les remarques ethnographiques développées dans ses écrits. C'est ainsi que sa vision de l'Égypte, tributaire de sa société de naissance, a été perçue par certains comme fragmentée, voire déformée. Or, elle souhaite au contraire faire connaitre le pays dans son ensemble et à plusieurs périodes de son histoire.

Entre condamnation et modération

Si les écrits d'Out-El-Kouloub tentent d'être davantage représentatifs de la société, les destinataires locaux sont-ils plus nombreux ? La question du lectorat rejoint « la notion d'efficacité ", ici appliquée au contexte littéraire. Dans Harem, un chapitre est consacré au katb al-kîtab (contrat de mariage) dans une famille cairote assez aisée. À l'instar de Balzac dans Le Contrat de mariage, elle veut aussi en donner la signification sociale, économique. Le futur conjoint est « un des maires les plus riches de la Haute Égypte». Il paye trois cents livres de dot et promet cent cinquante livres d'arrière-dot, somme garantie à la femme en cas de divorce. La mère de la mariée se réjouit « des volailles et des fruits dont son gendre sans doute la comblera ». La romancière, dans un style concis, veut tout dire, les cérémonies, les pratiques superstitieuses, les mentalités. Sur cette même question du mariage :

- La jeune fille pourtant ne paraîtra point. Elle ne quittera même pas sa chambre. Deux témoins iront recueillir de sa bouche le «oui» fatidique (...). Une vieille Circassienne (...) prodigue les recommandations :

- Surtout, ne réponds pas tout de suite, tu semblerais avoir hâte de quitter tes parents. Quand on t'aura posé par trois fois la question, alors prends la voix la plus douce et la plus humble pour dire : oui. ${ }^{21}$

La condamnation est ici sous-jacente. Out-El-Kouloub qui écrit dans une optique réaliste et modérée nous oblige à reformuler la notion de littérature engagée. Dans Ramza le combat est constant, bien qu'il n'y ait pas de véritable interpellation. Ainsi, le personnage est le symbole de l'Égyptienne révoltée qui ne renonce pas, pourtant 
lorsqu'elle rencontre une jeune fille, qui « trouve charmant le fils du drapier voisin » et redoute un mariage arrangé avec son cousin de vingt ans son aîné, « [elle] os[e] à peine lui conseiller de tenir tête à son père » et déclare : «ce sont des décisions qu'il faut prendre seule, si l'on se sent assez forte $»^{22}$.

Si aujourd'hui des aspects de son écriture peuvent apparaître traditionnels et conventionnels, c'est justement en raison de ce parti pris de modération, de prudence. Ces nuances systématiques permettent-elles l'efficacité d'un message féministe, parfois ténu ? La défense de certaines valeurs traditionnelles ne se fait-elle pas au détriment de l'expression féministe? Il s'agit, d'une part, de prouver que l'Égypte est riche de traditions, de dignité, de chaleur, et, d'autre part, de relever puis condamner les travers engendrés par ces éléments. Dans la mesure où Out-El-Kouloub incarne une génération d'intellectuels, désireuse de vivre sereinement deux cultures, deux types d'influences, n'est-ce pas là un projet chimérique ? Le paradoxe qui gagne de nombreux personnages est certes représentatif des chocs culturels, mais il semble également toucher l'auteur. Ainsi, le bilinguisme et le biculturalisme constituent des thèmes non négligeables de son roman le plus autobiographique Ramza. À travers un autre de ses personnages, la romancière aborde le danger d'abandonner sa culture au profit d'une autre, c'est un thème récurrent de son œuvre. «Mounira parlait le français mieux que l'arabe. » L'idéal est personnifié par Ramza qui maîtrise parfaitement plusieurs langues mais a commencé par l'étude de l'arabe classique.

Topographie des livres aimés

Les influences occidentales de ses références littéraires, de son écriture sont évidentes. Sans chercher à les recenser en totalité, on peut ici en relever les plus frappants. Au fil de son œuvre, par exemple, à travers ses personnages de femmes cultivées, Out-ElKouloub laisse entrevoir ses goûts en matière de littérature, une topographie des livres aimés se dessine. Elle éprouve " une véritable passion pour Mauriac », nous dit l'un de ses préfaciers ${ }^{23}$, "personne mieux qu'elle ne parle du Baiser au lépreux». Cette admiration se manifeste dans Nazira, l'un des trois Contes de l'amour et de la mort. Il ne s'agit pas pour autant $d^{\prime}$ « une littérature de décalcomanie »" le récit qui incrimine le patriarcat est égyptien, les pratiques superstitieuses décrites en font l'originalité. Aucun des récits n'est véritablement emprunt d'influences directes précises. Les goûts éclectiques de la romancière se traduisent par un aspect générique polymorphe. Ouvrage de maximes, genre très répandu en Orient, romans, contes, petites nouvelles, forment l'œuvre. Sur le style, les variations sont aussi nombreuses.

Dans Le Coffret hindou, une référence explicite est faite aux Désenchantées, le " roman des harems turcs contemporains » de Pierre Loti ${ }^{25}$. Ce renvoi emprunte aux réalités de début de siècle puisque Loti était lu et apprécié par l'élite égyptienne. L'identification se faisait naturellement avec le harem égyptien copié sur le modèle turc.

- Vous vous êtes nourrie des Désenchantées, lui dit-il enfin.

- J'ai lu en effet les Désenchantées quand j'avais seize ans (...). J'espère que vous me jugez assez intelligente pour n'en être pas restée à ce féminisme à l'eau de rose. Connaissez-vous Kassem Amin ? ${ }^{26}$

La question suggère que si l'élan vient de l'Europe, le changement doit se faire de l'intérieur. D'ailleurs, Out-El-Kouloub façonne à cette idée le personnage de Mademoiselle Hortense, l'institutrice française larmoyante de Ramza: «La pauvre Mademoiselle était certes tout le contraire d'une révoltée » mais « en vivant à ses côtés, en conversant avec elle, j'élargis mes horizons. Elle venait d'un autre monde, un monde 
de femme comme le mien, mais différent du mien, qu'elle me faisait connaître. $»^{27}$ Samiha, qui intervient dans le dialogue, a étudié à Paris, mais c'est en Égypte que sa révolte a pris forme et c'est le célèbre féministe égyptien Kassem Amin qu'elle cite en priorité. Elle-même a un livre en préparation sur le sujet de la libération féminine. Il présentera les revendications de la femme, "dès qu'elle aura acquis suffisamment d'autorité ».

Son argument majeur reflète le «raisonnement-prétexte» par lequel passent les progressistes de l'époque afin de préparer les mentalités : « Notre pays ne progressera pas vraiment tant que les femmes y seront maintenues dans l'ignorance et l'esclavage. $\aleph^{28}$ La justification du féminisme sera légitimée au plan national : elle sera reprise par de nombreux politiciens et en particulier par Nasser. En 1956, la Constitution accorde aux femmes le droit de vote et l'éligibilité29.

Mystification

31 Par ailleurs, les références littéraires des personnages révèlent de quelle manière l'auteur s'est jouée des représentations, des attentes du public français. Cela se manifeste dans les titres mêmes de ses écrits, Harem, Ramza, Zanouba, Hefnaoui le magnifique. Marqués par l'époque, ces noms sont destinés à éveiller la curiosité des lecteurs émoustillée par les récits de voyage, en flattant leur goût pour un Orient chatoyant. Ruse ludique d'auteur qui n'est pas s'en rappeler l'œuvre de Pierre Loti et le succès considérable rencontré lors de la sortie d'Aziyadé:

Parmi les étudiants de Sorbonne en 1906, le livre connut un grand écho: avec

l'enthousiasme de nos vingt ans, nous nous arrachions la Revue des Deux Mondes où paraissait le livre merveilleux. ${ }^{30}$

Dans un même temps, la romancière se gausse de ce féminisme " à l'eau de rose " et emploie le même type de stratagèmes, manifeste des goûts littéraires communs, n'excluant pas une bonne dose d'images, de tableaux exotiques. Elle amène ses différents témoignages en offrant également une part de rêve et d'évasion conformes à l'aspiration des lecteurs et fidèles à son amour de l'Égypte d'antan. Hormis la référence directe aux Désenchantées contenue dans Le Coffret hindou, des similitudes apparaissent entre ce roman de Loti et Ramza. On pourrait voir dans cette correspondance les mêmes conflits engendrés par des sociétés patriarcales semblables, mais les ressemblances entre l'héroïne d'Out-El-Kouloub et celles du romancier français nous autorisent à relever, même si elle s'en défend, l'inclination de la romancière pour l'œuvre de Loti. Si elle s'en cache, n'est-ce pas justement pour éviter le même type d'attaques? Ce jeu qu'elle instaure avec les lecteurs épaissit le climat mystérieux qui entoure son œuvre, sa vie. Les rumeurs les plus saugrenues courent sur son compte, la plus surprenante remettant en cause l'authenticité de ses écrits.

Conclusion

33 Fille d'un shaykh de confrérie mystique, Out-El-Kouloub rend hommage à son père: « [II] a su dans sa sagesse, développer en moi l'amour de nos traditions, sans me priver des bienfaits d'une éducation moderne. ${ }^{31}$ Dans cette optique, elle tente de concilier deux systèmes de valeurs parfois diamétralement opposés et cette dichotomie se retrouve dans son œuvre. Elle transmet l'idée de la difficulté de vivre sereinement deux cultures, mais aussi son sentiment de piété à l'égard de l'autrefois, de l'esprit de tradition qui se manifeste dans les moindres décisions, au niveau du quotidien de ses personnages, car «du passé, sont participantes et gardiennes des valeurs métaphysiques auxquelles on doit trop pour pouvoir les circonscrire ou les 
domestiquer $»^{32}$. «Madame Out-El-Kouloub ne se croit pas obligée de blâmer avec des mines d'hypocrite tout le passé de l'Orient. $»^{33}$ Implicitement, la culture intellectuelle représente une échappatoire, mais aussi une issue, toujours dans son optique de discernement, de retenue. Cette attitude ne surprendrait pas, si Out-El-Kouloub ne mettait en scène des personnages passionnés qui invalident presque totalement son apologie de la modération. Du point de vue de l'un de ses critiques, l'auteur a adopté une attitude "de sagesse dictée par l'histoire elle-même, une volonté de prendre et de pardonner, une acceptation sans amertume des choses fatales ${ }^{34}$. Mais le conflit entre l'ancien et le moderne est âpre, il n'en finit pas de nous dévoiler toutes les contradictions d'une œuvre littéraire, d'un lieu, d'une époque.

\section{BIBLIOGRAPHIE}

Bibliographie d'Out-El-Kouloub

Au hasard de la pensée, Le Caire, Al Maaref, 1934, 162 pages.

Harem, préface de Paul Morand, Gallimard, Paris, 1937, 238 pages.

Trois contes de l'amour et de la mort, préface d'André Maurois, Corrêa, Paris, 1940, 313 pages.

Zanouba, préface de Jérôme et Jean Tharaud, Gallimard, Paris, 1947,222 pages.

Le Coffret hindou, préface de Jean Cocteau, Gallimard, Paris, 1951, 226 pages.

La Nuit de la Destinée, préface d'Emile Dermenghem, Gallimard, Paris, 1954, 212 pages.

Ramza, préface d'Henri Guillemin, Gallimard, Paris, 1958, 254 pages.

Hefnaoui le magnifique, préface d'Henri Peyre, Gallimard, Paris, 1961, 218 pages.

\section{NOTES}

1. La première nouvelle d'Albert Cossery, Un homme supérieur, paraît deux ans après, dans le numéro de Noël 1936 de La Semaine égyptienne.

2. Luizard Pierre-Jean, « Le Soufisme égyptien contemporain », Égypte/Monde arabe $\mathrm{n}^{\circ} 2$, CEDEJ, Le Caire, 1990, p. 71.

3. Andrée Chédid quitte l'Égypte en 1945, Albert Cossery en 1946.

4. Si Out-El-Kouloub se tait désormais, les deux écrivains en exil volontaire depuis de nombreuses années, affirment porter en eux une Égypte qui ne les quitte pas. Le pays natal est ainsi terre rêvée, reformulée, muse, Cossery se plaisait à dire : « Je ne me suis jamais senti hors de l'Égypte. Je la porte en moi. Et même des détails vus ou entendus il y a cinquante ans, je peux les mettre dans un nouveau récit. Ils me sont restés en mémoire. ", Mitrani Michel, Conversations avec Albert Cossery, Éditions Joëlle Losfeld, Paris, 1995, p. 8.

5. Viatte Auguste, Histoire comparée des littératures francophones, Nathan, Paris, 1981, p. 95. 
6. Elle publie huit écrits de 1934 à 1961, la quasi-totalité chez Gallimard.

7. Peyre Henri, préface à Ramza d'Out-El-Kouloub, p. 7.

8. Joubert Jean-Louis, «Écritures arabes en français », Les cahiers de l'Orient $n^{\circ} 4$, SFEIR, Paris, 1986, p.174.

9. Deniau Xavier, La Francophonie, Que sais-je ?, PUF, Paris, $1^{\text {re }}$ édition 1983, $3^{\mathrm{e}}$ édition janvier 1995, p. 18.

10. Out-El-Kouloub, Ramza, p. 96.

11. L'Égyptienne, La Déclaration, $1^{\text {re }}$ année, $n^{\circ} 1$, Le Caire, février 1925, p. 3.

12. Abou Sélim, «Francophonie: les leçons libanaises ", Les Cahiers de l'Orient, $\mathrm{n}^{\circ} 4$, SFEIR, Paris, 1986, p. 161. Sur ces questions francophones, voir également, du même auteur, Le Bilinguisme arabe-français au Liban, PUF, Paris, 1962.

13. Hussein Taha, Fusûl fi-l-adâb wa-l-naqd, Dar El Maaref, Le Caire, 1969, p. 61, traduction de Sonia Rezk Ghattas in Visages de femmes égyptiennes, Université de Californie, 1979, p. 57.

14. Laroui Abdallah, L'Idéologie arabe contemporaine, Maspero, Paris, 1967, p.182.

15. Titre de l'un des trois contes.

16. Trois Contes de l'amour et de la mort par Out-El-Kouloub, critique de Josée Sékaly dans la revue Images, $\mathrm{n}^{\circ}$ 578, octobre 1940, p. 13.

17. Luthi Jean-Jacques, Introduction à la littérature d'expression française en Égypte (de 1798 à 1945), Éditions de l'École, Paris, 1974, p. 15.

18. Ibid, p. 257.

19. Union des femmes égyptiennes.

20. Fenoglio-Abd El Aal Irène, «Au début d'une expression féminine ». Défense et illustration de l'Égyptienne, CEDEJ, Le Caire, 1988, p. 116.

21. Ramza, p.60-61.

22. Ibid., p. 226.

23. Maurois André, préface des Trois Contes de l'amour et de la mort, Corrêa, Paris, 1940, p. 9. Le titre est évidemment une référence à Tolstoï.

24. La formule est d'Irène Fenoglio Abd el Aal.

25. Loti Pierre, Les Désenchantées, « Roman des harems turcs contemporains », 1906 pour la première édition, Omnibus, Presses de la Cité, Paris, 1989.

26. Out-El-Kouloub, Le Coffret hindou, Gallimard, Paris, 1951, p. 143.

27. Ramza, p. 92-93.

28. Ibid, p. 144.

29. Le 29 septembre 1952, année de la révolution égyptienne, le droit de vote est accordé aux femmes qui savent lire et écrire, des réserves sont faites sur l'éligibilité. 30. M. Grosdidier de Matons cité par Alain Quella-Villéger dans Pierre Loti l'incompris, Presses de la Renaissance, Paris, 1986, p. 251.

31. Dédicace faite à son père dans la Nuit de la destinée.

32. Berque Jacques, "Société et Lettres Arabes contemporaines ", Cahiers Internationaux de Sociologie, Paris, 1964, p. 23.

33. Article de Gabriel Bounoure sur Ramza, d'Out-El-Kouloub, dans La Revue du Caire, n²26, juin1959, p. 301.

34. Ibid. 
INDEX

Mots-clés : littérature, francophonie, féminisme, orientalisme 